\title{
misonity
}

Revista Educación

ISSN: 0379-7082

ISSN: 2215-2644

revedu@gmail.com

Universidad de Costa Rica

Costa Rica

\section{Factores de riesgo en estudiantado universitario cuyos progenitores abusan de bebidas alcohólicas}

\author{
Alvarado Cordero, Silvia Elena; Frías Quesada, Carmen María \\ Factores de riesgo en estudiantado universitario cuyos progenitores abusan de bebidas alcohólicas \\ Revista Educación, vol. 42, núm. 2, 2018 \\ Universidad de Costa Rica, Costa Rica \\ Disponible en: http://www.redalyc.org/articulo.oa?id=44055139026 \\ DOI: https://doi.org/10.15517/revedu.v42i2.25760
}

Esta obra está bajo una Licencia Creative Commons Atribución-NoComercial-SinDerivar 3.0 Internacional. 
Factores de riesgo en estudiantado universitario cuyos progenitores abusan de bebidas alcohólicas

\author{
Risk Factors in University Students Whose Parents Abuse Alcohol
}

Silvia Elena Alvarado Cordero [1]

DOI: https://doi.org/10.15517/revedu.v42i2.25760

Universidad de Costa Rica, Costa Rica

Redalyc: http://www.redalyc.org/articulo.oa?id=44055139026

sil.alvarado@gmail.com

Carmen María Frias Quesada [2]

Universidad de Costa Rica, Costa Rica

carmen.frias@ucr.ac.cr

Recepción: 26 Julio 2016

Aprobación: 25 Abril 2018

\title{
Resumen:
}

Este artículo presenta los principales hallazgos de la investigación "Factores de riesgo psicosociales en población universitaria", realizada con estudiantes de la Sede Rodrigo Facio de la Universidad de Costa Rica. Se estudiaron los factores de riesgo: ansiedad, codependencia, baja autoestima y depresión, y se relacionaron con la condición de ser persona adulta cuyos progenitores presentaban abuso de bebidas alcohólicas. La investigación fue planteada en colaboración con personal funcionario de la Universidad de Puerto Rico, Recinto Río Piedras. Esta fue de tipo correlacional y para recopilar la información se aplicó un cuestionario en línea, el cual, en su versión inicial, fue el utilizado en la investigación realizada en Puerto Rico y cuyas variables fueron los factores de riesgo objeto de estudio. Participaron 2009 estudiantes, que de manera voluntaria y en condiciones de anonimato y confidencialidad estuvieron de acuerdo en completar el cuestionario. Los resultados muestran que el grupo de participantes se caracteriza, en su mayoría, con probabilidades de codependencia, síntomas de depresión entre leves y moderados, con ausencia de síntomas de ansiedad y una autoestima normal y alta. No se encontraron diferencias estadísticamente significativas en ninguno de los factores de riesgo estudiados, entre los hijas e hijos de progenitores con abuso de alcohol (HADA) y aquellos progenitores que no lo presentan (NO HADA). El estudio aportó información que puede contribuir en la comprensión de los factores de riesgo de la población universitaria y para proponer acciones que contribuyan a su enfrentamiento desde el quehacer de la profesión de orientación.

Palabras Clave: Factores de riesgo, codependencia, autoestima, ansiedad, depresión, alcoholismo.

\section{AbstraCt:}

This article aims to present the main findings of the investigation "Psychosocial Risk Factors in a University Population", conducted with students from the Rodrigo Facio Campus of the University of Costa Rica. Risk factors such as anxiety, codependency, low self-esteem, and depression were studied and related to the presence of characteristics of being an adult and the condition of parents who abused alcohol. The research was carried out in conjunction with staff members from the University of Puerto Rico, Río Piedras Campus. The study was correlational and in order to gather information, an online questionnaire was completed. The initial version of the questionnaire was used in a research carried out in Puerto Rico, whose variables were the risk factors studied in this research. Voluntarily and under conditions of anonymity and confidentiality, two thousand nine students agreed to answer the questionnaire. Results show that most of the participants can be characterized as having a probability of codependency, symptoms of mild to moderate depression, no symptoms of anxiety and average and high self-esteem. No statistically significant differences in any of the factors studied were found between adult children of alcoholic parents and those of non-

\section{Notas de AUtor}

[1] Orientadora, con un Doctorado en Educación, con énfasis en Mediación Pedagógica, Actualmente labora como docente de la Escuela de Orientación y Educación Especial, es investigadora activa en el Instituto de Investigaciones en Educación y docente en la Maestría en Educación con énfasis en Orientación laboral. Es lectora y directora de trabajos finales de graduación. Anteriormente laboró por casi 15 años con el Ministerio de Educación Pública, como orientadora en Equipos Interdisciplinarios y con Departamentos de Orientación de Secundaria.

[2] Orientadora y con un posgrado en Evaluación Educativa de la Universidad de Costa Rica. Profesora asociada, jubilada de la Escuela de Orientación y Educación Especial. Directora de trabajos finales de graduación. 
alcoholic parents. The findings of this study provide information that contributes to the understanding of the risk factors in university student population, and to propose actions to face them during counselling interventions.

KEYWORDS: Risk factors, Co-dependency, Self-esteem, Anxiety, Depression, Alcoholism.

\section{INTRODUCCIÓN}

Este artículo tiene por objetivo presentar los principales resultados obtenidos de la investigación "Factores psicosociales de riesgo en estudiantes universitarios de Costa Rica", realizada en conjunto con miembros del Departamento de Consejería para el Desarrollo Estudiantil de la Universidad de Puerto Rico, Recinto de Río Piedras. La investigación se propuso analizar los factores de riesgo de la población estudiantil de la Sede Rodrigo Facio de la Universidad de Costa Rica. Los factores de riesgo incluidos en este estudio fueron: ansiedad, codependencia, baja autoestima y depresión, los cuales se relacionaron con la condición de ser persona adulta, cuyos progenitores presentaban abuso de bebidas alcohólicas.

La ansiedad, codependencia, baja autoestima y depresión constituyen factores de riesgo y como lo plantean Ramírez y De Andrade (2005) se pueden considerar situaciones personales o sociales, que incrementan la probabilidad de que la persona inicie el consumo, en este caso de bebidas alcohólicas. Es decir, los factores de riesgo traen consigo un aumento en la probabilidad de que se presente determinada conducta, pero que además incide negativamente en el desarrollo y bienestar de la persona.

Para el análisis de estos factores de riesgo cobra especial importancia el papel de la familia como grupo primario de socialización. Como lo plantea Parada (2010), la familia es la institución que determina tanto a la sociedad como a las personas, en cuanto asume las funciones de la procreación y crianza de la descendencia, así como funciones económicas, culturales, educativas, religiosas entre otras. Este autor reitera que "la familia es la transmisora de la vida, pero también de los puntos de referencia global para la vida” (p. 19) Aunque no es posible atribuir todos los factores de riesgo al entorno familiar, si es posible que este en algunos casos lo sea.

La familia no está aislada de la sociedad en la que se encuentra, más bien, forma parte de esta y sus problemas psicosociales se reflejan en ella. En Costa Rica, Bejarano (2012) señala que las investigaciones nacionales han establecido el abuso de alcohol aproximadamente en un $40 \%$ de las personas; por su parte, la encuesta nacional realizada en el año 2010 establece la edad promedio de inicio del consumo en 17,4 años, con una edad de comienzo significativamente mayor para las mujeres (18,5 años) en comparación con la de los hombres ( 16,6 años).

Las familias del estudiantado universitario no escapan a estas realidades y es así como también a este grupo lo alcanzan las consecuencias de la exposición a situaciones como las mencionadas anteriormente. Sus experiencias vitales arrancaron en el seno de la familia donde nacieron y es allí donde se han sentado las bases de su desarrollo.

A eso se suma que una gran parte de la formación universitaria ocurre cuando el estudiantado se encuentra en la etapa de la adolescencia o bien dejándola atrás para dar paso al inicio de la adultez, que es cuando se espera que asuma cada vez más responsabilidades consigo mismo y con su proyecto de vida.

La cotidianidad para cada estudiante en la universidad es compleja, producto de las altas demandas que enfrenta en su avance académico y a la necesidad de contar con recursos personales y externos para hacerles frente de manera satisfactoria.

Los factores de riesgo, además de poner en peligro el éxito de los estudios universitarios y el logro de la meta académica que se ha propuesto cada estudiante como parte de su proyecto de vida, pueden afectar su bienestar integral, incluidas las relaciones interpersonales que establece y la futura inserción laboral. Estas son razones que hacen importante su estudio, para que eventualmente las instancias universitarias encargadas de la vida estudiantil puedan proponer acciones tendientes a la atención de las poblaciones estudiantiles, para disminuir, de esa forma, el impacto de los factores de riesgo, para aumentar las probabilidades de concluir con éxito su carrera universitaria. 


\section{FUNDAMENTOS TEÓRICOS}

A continuación, se plantean los postulados teóricos del estudio. Cabe mencionar que los factores de riesgo se definen, de acuerdo con Manrique (2017, p. 17), como:

Cualquier rasgo, característica o exposición de un individuo que aumente su probabilidad de sufrir una enfermedad o lesión... Por el contrario, los factores protectores hacen alusión a las características y rasgos del individuo que disminuyen o eliminan la probabilidad de sufrir una lesión o enfermedad.

Seguidamente se desarrollan los cuatro factores de riesgo, objeto de la investigación: ansiedad, codependencia, baja autoestima y depresión.

Con respecto al factor ansiedad, todas las personas la experimentan en algún grado, debido a que es considerada una respuesta común de los seres humanos que pretende la adaptación. Por ejemplo, en el caso del estudiantado, situaciones como cumplir con plazos de entrega ajustado, ya sea para presentar una prueba o la entrega de un trabajo, demandan de un cierto grado de ansiedad que le permite cumplir con la demanda externa, de ahí que sea necesaria en la vida diaria. Sin embargo, esta ansiedad hay que distinguirla de otra que puede ser negativa. Al respecto, Sierra, Ortega y Zubeidat (2005, p.16) afirman que "la ansiedad patológica se manifiesta con mayor frecuencia, intensidad y persistencia que la ansiedad normal, es decir, presenta diferencias cuantitativas respecto a aquella".

Una de las características de la ansiedad consiste en que es anticipatoria de peligros o amenazas futuras; se presenta sin que exista una amenaza externa que pueda ser identificada, de ahí que tales amenazas son difusas e indefinidas. Entre las consecuencias de la ansiedad se encuentran la fatiga, la falta de motivación, iniciativa e interés.

Los trastornos afectivos han llegado a ser un problema de salud pública; en el caso de la población universitaria, la depresión es el síntoma más frecuente y que afecta el desarrollo de las personas a lo largo de la vida (Franck y De Raedt, 2007). Esta forma parte de los llamados trastornos afectivos, como lo señalan Riveros, Hernández y Rivera (2007), tienen por característica la alteración de la capacidad afectiva, la presencia de tristeza y afectan la capacidad de dar y recibir afecto.

Los estudios universitarios implican un ritmo de vida acelerado, debido a las demandas que suponen y a la existencia de un ambiente competitivo en el cual las personas deben mostrar un desempeño que les permita permanecer y avanzar en sus estudios. A eso se suma que el paso de un año a otro trae consigo cambios y, como lo plantea Rodríguez (2008), estas condiciones pueden resultar en un incremento en el número de personas con sentimientos de inestabilidad, tensión y malestar, que de mantenerse en el tiempo, pueden afectar el bienestar y calidad de vida de la persona que los experimenta. Así mismo, Rodríguez (2008, p.19) agrega que "en gran medida, esta tensión o malestar es debida a la percepción de las demandas situacionales (la interpretación que el sujeto hace de las mismas), la valoración de los recursos que posee para afrontarlas y los resultados de sus actuaciones".

En relación con la codependencia como factor de riesgo, se caracteriza por la presencia y convivencia en el grupo familiar de una persona encargada de la crianza, como el padre, la madre o ambos, que experimenta una adicción a las bebidas alcohólicas. Es claro que no solo la persona alcohólica padece la enfermedad, sino que se extiende al grupo familiar, que también desarrolla características similares a la persona que la sufre. $\mathrm{Al}$ respecto, la organización Codependientes Anónimos (s. f.) señala algunos patrones de comportamiento de las personas codependientes, como son la negación, baja autoestima, sumisión, complacencia, conformidad, control y evitación.

Además, se podría agregar que las personas que han desarrollado codependencia pueden presentar manifestaciones emocionales como miedo, tristeza, odio, coraje, resentimientos e inseguridades.

Por su parte, la autoestima cumple un doble papel, el de factor protector pero también como factor de riesgo. Los diferentes planteamientos teóricos coinciden en que una autoestima alta es un factor protector. 
Al respecto Branden (1995) menciona que una autoestima alta permite a la persona sentirse con confianza en su capacidad y valor como persona. Sin embargo, como plantean Cáceres, Salazar, Varela y Tovar (2006), los resultados de investigaciones no son concluyentes por cuanto algunas han planteado que una alta autoestima puede provocar que las personas se sientan confiadas y asuman comportamientos de riesgo.

Por otra parte, una baja autoestima se convierte en un factor de riesgo, porque como señalan Gonzales y Guevara (2016), hace sentir a la persona inapropiada, asume la creencia de no merecer cosas buenas y provoca que tome decisiones incorrectas. Adicionalmente, como lo señalan Franck y De Raedt (2007), existen investigaciones que han dado evidencia de que una baja autoestima puede estar asociada con la vulnerabilidad a la depresión. Por su parte, Naranjo (2007) agrega que la autoestima baja puede limitarse en un período relativamente corto, pero en otros casos podría prolongarse en el tiempo y, con ello, acarrear dificultades que impactan la vida de la persona; y que cuando coincide con otros eventos significativos como dificultades académicas, depresión y ansiedad, las consecuencias negativas pueden ser mucho más intensas.

Además de los cuatro factores de riesgo que se analizan en este estudio, se requiere retomar la condición familiar del estudiantado porque la familia es el agente encargado del proceso de socialización, se atienden las necesidades propias del desarrollo humano, también es donde están las bases de la personalidad y de las habilidades de interacción social.

En la bibliografía consultada, la familia donde al menos uno de los progenitores muestra una condición de abuso de alcohol, se cataloga como una familia alcoholizada. El abuso se define como "un patrón desadaptativo de utilización de una droga el cual puede manifestarse por consecuencias adversas, expresadas durante un período de 12 meses. También se determina que existe abuso cuando la persona ingiere cinco o más tragos por ocasión" (Bejarano, 2012, p.29).

Aunque las familias donde hay abuso de alcohol por parte de uno de sus progenitores parecen funcionar adecuadamente, Guzmán (2006, en Alazraki, 2006) plantea que es posible que alguna parte de sus miembros desarrollen características como la sobreprotección, establecimiento de alianzas excesivas en la familia, dificultad para la resolución de los conflictos y una rigidez extrema. De esta manera, el sistema familiar establece un estilo de vida que contribuye a que la enfermedad del alcoholismo continúe de generación en generación.

Según Valverde y Pochet (2006), las hijas y los hijos que provienen de un ambiente en el cual al menos uno de sus progenitores ha abusado del alcohol, tienden a mostrar conductas que reproducen los síntomas presentados por las personas adictas, por ejemplo, el uso de la negación respecto a lo que les molesta o afecta, como un mecanismo de sobrevivencia.

Las hijas y los hijos de personas alcohólicas se afectan profundamente por la enfermedad de alguno de sus progenitores y como lo señala Alazraki (2006), tienen una probabilidad cuatro veces mayor que otras personas de convertirse en alcohólicas. Además, con frecuencia se les dificulta la identificación y expresión de sus sentimientos, podrían ser muy rígidas y buscan ejercer control sobre las otras personas o ser dependientes de ellas, sienten que no tienen posibilidades de tomar decisiones con respecto a su forma de vida; con frecuencia se muestran deprimidas y sin capacidad de establecer relaciones de intimidad. Es así como estas características repercuten en la vida profesional y personal de quienes han tenido progenitores alcohólicos. Como lo explican Jiménez, Serra, Villafañe y Jiménez (2011, p.13) el consumo abusivo de bebidas alcohólicas en un miembro de la familia afecta a estudiantes en aspectos como "las relaciones sociales en la universidad, familia y sociedad, manejo de sus relaciones de pareja y un impacto adverso en su proceso académico pudiendo afectarse su éxito educativo, vocacional y eventualmente presentar dificultad en su desempeño laboral”.

\section{Procedimiento metodológico}

Se realizó un estudio correlacional que "...tiene como finalidad conocer la relación o grado de asociación que exista entre dos o más conceptos, categorías o variables en un contexto en particular.” (Hernández, 
Fernández y Baptista; 2008, p.81). Es decir, en el caso de esta investigación, los resultados describen la relación entre las variables factores de riesgo en la población participante y la condición de ser persona adulta cuyos progenitores presentaban abuso de bebidas alcohólicas.

Para efectos del estudio se consideraron como variables los factores de riesgo: codependencia, depresión, ansiedad y autoestima (dependientes), así como el ser hija o hijo con al menos uno de los progenitores con abuso de bebidas alcohólicas (independiente).

La información se recopiló mediante un cuestionario, el cual fue administrado a una muestra no probabilística, de estudiantes que de manera voluntaria accedieron a participar. Se obtuvo una respuesta de 2009 estudiantes de la Universidad de Costa Rica, Sede Rodrigo Facio, pertenecientes a todas las áreas de estudio: Bellas Artes, Ciencias Sociales, Ciencias Básicas, Ingeniería, Salud y Ciencias Agroalimentarias.

La participación de estudiantes fue voluntaria y por disponibilidad dentro de los parámetros de anonimato y confidencialidad.

\section{Instrumento}

El instrumento utilizado en este estudio se elaboró a partir de la investigación de Jiménez, Serra, Villafañe y Jiménez (2011), titulada "Hijos adultos de madres/padres alcohólicos y factores de riesgo psicológicos en estudiantes universitarios”, realizada con estudiantado de la Universidad de Puerto Rico, Recinto de Río Piedras.

La primera parte del instrumento solicitaba información sociodemográfica y en la segunda se incluyeron 38 preguntas que incluían las siguientes cinco subescalas:

- Sub-escalas de ansiedad y depresión de la Lista de cotejo de síntomas-36 (LCS-36) (Derogatis y Cleary, 1977; McNeil, Greenfield, Attkinson, y Binder, 1989) la cual ha sido utilizada con la población puertorriqueña (Bernal, Bonilla, y Santiago, 1995). La sub-escala de ansiedad tiene siete (7) reactivos y la de depresión nueve (9).

- La Escala de autoestima Rosenberg (Rosenberg, 1965) que consta de diez (10) reactivos ha sido traducida al español y validada en poblaciones de habla hispana (Martin-Albo, Núñez, Navarro, y Grijalvo, 2007).

- La sub-escala rasgos de codependencia utiliza dos reactivos de cada uno de los tres factores del instrumento codependencia (Pérez-Gómez y Delgado-Delgado, 2003) los cuales evaluaron las siguientes áreas: no afrontamiento, focalización en el otro y necesidad compulsiva de ayuda.

- Sub-escala Hijos adultos de padre o madre alcohólico (HADA) se utilizó la versión corta del Children of Alcoholics Screening Test (CAST) (Hodgins, Maticka-Tyndale, El-Guebaly y West, 1993) la cual consta de seis (6) reactivos. Este instrumento hace referencia a padre, madre o encargado a cargo de la crianza del estudiante o la estudiante. Una respuesta positiva a tres o más de los reactivos se considera que identifica a una persona como un HADA.

Como el instrumento fue diseñado para el contexto estudiantil de la Universidad de Puerto Rico, fue necesario realizar un proceso de revisión y análisis para adecuarlo al costarricense. Para ello, se sometió al criterio de seis profesionales en el ejercicio de la orientación, a quienes se les solicitó que valoraran el planteamiento de cada ítem en función del contexto y la idiosincrasia del estudiantado de la Universidad de Costa Rica.

Una vez recibidas las sugerencias fueron incorporadas al cuestionario. Cumplida esa fase se envió en formato digital a un grupo universitario de estudiantes, con el propósito de realizar una prueba piloto, luego de esta aplicación fueron incluidas las correcciones necesarias. 
Las principales modificaciones realizadas al cuestionario fueron: los ítems se redactaron en segunda persona singular (usted), se incorporó lenguaje inclusivo de género y algunos cambios de redacción, de términos y del formato de la presentación de las preguntas.

La aplicación del instrumento se realizó vía web y se dio un plazo de espera de respuestas por parte del estudiantado de 30 días. Se le remitió a la población estudiantil participante del estudio la invitación para completar el cuestionario y el enlace donde se ubicaba este. Al completar el instrumento las respuestas se almacenaron automáticamente en una base de datos.

El análisis estadístico de los datos se realizó utilizando el programa Statistical Package for Social Sciences (SPSS), versión 17.0 para Windows. Para el análisis de los datos se obtuvieron frecuencias absolutas y relativas; desviaciones estándar, pruebas t student, con el propósito de determinar diferencias estadísticamente significativas entre el estudiantado universitario en función de las variables incluidas en este estudio.

\section{Principales Resultados}

El instrumento fue contestado por 2009 estudiantes por disponibilidad, de los cuales 62\% eran mujeres y el 38\% hombres. Las edades del estudiantado participante se distribuyeron de la siguiente manera: un $45 \%$ entre los 20 y 23 años; un 30\% entre 16 y 19 años y un 18\% en el grupo de 24 a 27 años y solamente un 7\% tenía entre 28 y más años. Los resultados evidencian que las tres cuartas partes del estudiantado se ubican entre los 16 y 23 años de edad.

Con respecto al año de estudios en que se encontraban, un $44 \%$ estaba entre primero y segundo y $37 \%$ en tercero y cuarto. En cuanto a las carreras participantes, un 33\% procedía de Ciencias Económicas y $24 \%$ de Ciencias Sociales. En la consulta de con quien creció la mayor parte de su infancia, se encontró que un 44\% con padre y madre y un $42 \%$ respondieron que creció solo con la madre.

En la siguiente tabla se presentan los resultados obtenidos en relación con los factores de riesgo: síntomas de depresión y ansiedad, autoestima y rasgos de codependencia. 
TABLA 1

Distribución del estudiantado participante según factores de riesgo Sede Rodrigo Facio, Universidad de Costa Rica, 2012

\begin{tabular}{|c|c|c|}
\hline Factores de riesgo & $\mathbf{N}$ & Porcentaje \\
\hline \multicolumn{3}{|c|}{ Codependencia } \\
\hline Sin codependencia & 719 & $36 \%$ \\
\hline $\begin{array}{l}\text { Con probabilidad de } \\
\text { codependencia }\end{array}$ & 1290 & $64 \%$ \\
\hline \multicolumn{3}{|c|}{ Depresión } \\
\hline No hay síntomas & 630 & $31 \%$ \\
\hline Leve & 599 & $30 \%$ \\
\hline Moderado & 495 & $25 \%$ \\
\hline Severo & 285 & $14 \%$ \\
\hline \multicolumn{3}{|c|}{ Ansiedad } \\
\hline No hay síntomas & 1333 & $66 \%$ \\
\hline Leve & 489 & $24 \%$ \\
\hline Moderado & 163 & $8 \%$ \\
\hline Severo & 24 & $1 \%$ \\
\hline \multicolumn{3}{|c|}{ Autoestima } \\
\hline Baja & 251 & $12 \%$ \\
\hline Moderada & 270 & $13 \%$ \\
\hline Normal o alta & 1488 & $74 \%$ \\
\hline
\end{tabular}

Nota: Fuente cuestionario "Factores de riesgo psicosociales en población universitaria", 2012.

En la Tabla 1 se presentan los factores de riesgo, a saber: codependencia, depresión, ansiedad y autoestima. Con respecto al factor codependencia, un $64 \%$ de la población estudiantil tiene probabilidades de presentarla, no así el 36\%. En cuanto al factor depresión, un 31\% no presenta síntomas; el 55\% presenta síntomas considerados entre leves y moderados; mientras un $14 \%$ presenta los síntomas de manera severa. 
En el factor ansiedad solamente un 1\% presenta síntomas severos; un 66\% no los presenta y el 32\% sí muestra síntomas que se pueden considerar leves y moderados.

En relación con el factor autoestima, casi las tres cuartas partes de la población estudiantil presenta una autoestima entre normal y alta. Un $12 \%$ del estudiantado posee una autoestima baja.

Con el propósito de determinar si existían diferencias significativas entre los hombres y mujeres, se aplicó la prueba t student, y los resultados se pueden observar en la Tabla 2.

TABLA 2

Diferencias por sexo en los factores de riesgo. Prueba $t$

student. Sede Rodrigo Facio, Universidad de Costa Rica, 2012

\begin{tabular}{lcccccc}
\hline \multicolumn{7}{c}{ Grupo } \\
\hline & Hombres & \multicolumn{7}{c}{ Mujeres } \\
\hline & Media & DS & Media & DS & $t$ & dff \\
\hline Ansiedad & 10,7 & 4,3 & 11,8 & 4,8 & $-5,3^{* * *}$ & 2007 \\
\hline Codependencia & 4,1 & 1,4 & 3,9 & 1,5 & $3,7^{*}$ & 2007 \\
\hline Autoestima & 32,7 & 5,9 & 32,7 & 5,7 & 0,0 & 2007 \\
\hline Depresión & 20,1 & 7,8 & 22,8 & 8,5 & $-7,0^{* *}$ & 2007 \\
\hline${ }^{*} p<.05 ;{ }^{* \star} p<.01$ & & & & & &
\end{tabular}

Nota: Fuente cuestionario "Factores de riesgo psicosociales en población universitaria", 2012.

La Tabla 2 muestra las diferencias por sexo en los factores de riesgo emocional. Los factores ansiedad, depresión y codependencia muestran diferencias significativas entre hombres y mujeres. Estas últimas obtienen resultados que evidencian mayor presencia de síntomas de ansiedad y depresión. Por su parte, el grupo de hombres participantes muestra una mayor codependencia comparado con las mujeres. No se encontraron diferencias significativas entre hombres y mujeres en el factor de autoestima.

En relación con la distribución del estudiantado de acuerdo con el sexo, se obtienen los siguientes resultados en la Figura 1. 


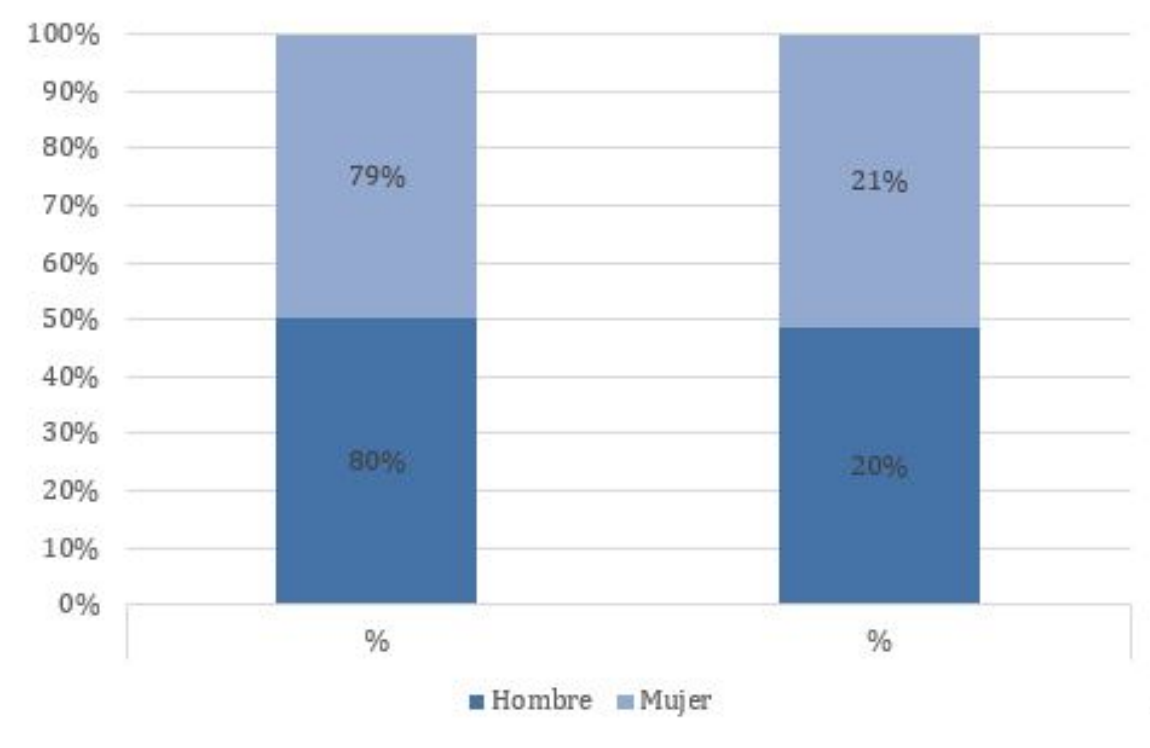

FIGURA 1

Distribución del estudiantado participante por sexo, según condición HADA y No HADA. Sede Rodrigo Facio, Universidad de Costa Rica, 2012 Nota: Fuente cuestionario “Factores de riesgo psicosociales en población universitaria”, 2012.

El $80 \%$ de la población masculina y el $79 \%$ de la población femenina no presentan características de hijos adultos de padres alcohólicos (HADA); por su parte un $20 \%$ de los hombres y un $21 \%$ de las mujeres sí presentan tales características. Es decir, para el total de la población solamente un $20,5 \%$ presenta características de HADA.

De acuerdo con el sexo, no se encontraron diferencias estadísticamente significativas entre el estudiantado con características HADA.

A continuación, se presentan los resultados de la distribución del estudiantado según los factores de riesgo ansiedad, codependencia, autoestima y depresión y la condición de ser HADA y NO HADA (Tabla 3).

TABLA 3

Distribución del estudiantado participante según factor de riesgo ansiedad y condición de ser HADA Y No HADA. Sede Rodrigo Facio, Universidad de Costa Rica, 2012

\begin{tabular}{l|c|c|c|c|c|c}
\hline \multirow{2}{*}{$\begin{array}{c}\text { Factor } \\
\text { ansiedad }\end{array}$} & \multicolumn{2}{c}{ No HADA } & \multicolumn{2}{c|}{ HADA } & \multicolumn{2}{c}{ Total } \\
\cline { 2 - 7 } $\begin{array}{l}\text { No hay } \\
\text { sintomas }\end{array}$ & 1074 & $67 \%$ & 63 & $63 \%$ & 1333 & $66 \%$ \\
\hline Leve & 382 & $24 \%$ & 26 & $26 \%$ & 489 & $24 \%$ \\
\hline Moderado & 123 & $8 \%$ & 10 & $10 \%$ & 163 & $8 \%$ \\
\hline Severo & 20 & $1 \%$ & 1 & $1 \%$ & 24 & $1 \%$ \\
\hline
\end{tabular}

Nota: Fuente cuestionario "Factores de riesgo psicosociales en población universitaria", 2012.

En relación con el factor de riesgo ansiedad, las frecuencias resultantes en los grupos HADA Y NO HADA tienden a ser semejantes. Con respecto al grupo total, dos terceras partes pueden ubicarse en la categoría sin 
síntomas, mientras que casi una cuarta parte se ubica en el grupo de ansiedad leve y solo un $9 \%$ se encuentra en las categorías de moderado y severo.

TABLA 4

Distribución del estudiantado participante según factor de riesgo codependencia y condición de ser HADA Y NO HADA. Sede Rodrigo Facio, Universidad de Costa Rica, 2012

\begin{tabular}{lcccccc}
\hline \multirow{2}{*}{$\begin{array}{c}\text { Factor } \\
\text { codependenci } \\
\text { a }\end{array}$} & \multicolumn{2}{c}{ NO HADA } & \multicolumn{2}{c}{ HADA } & \multicolumn{2}{c}{ Total } \\
\cline { 2 - 7 } & $\mathrm{N}$ & $\begin{array}{c}\text { Frecuenci } \\
\text { a relativa }\end{array}$ & $\mathrm{N}$ & $\begin{array}{c}\text { Frecuencia } \\
\text { relativa }\end{array}$ & $\mathrm{N}$ & $\begin{array}{c}\text { Frecuencia } \\
\text { relativa }\end{array}$ \\
\hline $\begin{array}{l}\text { Sin } \\
\text { codependencia }\end{array}$ & 583 & $36 \%$ & 136 & $33 \%$ & 719 & $36 \%$ \\
\hline $\begin{array}{c}\text { Con probabilidad } \\
\text { de codependencia }\end{array}$ & 1016 & $64 \%$ & 274 & $67 \%$ & 1290 & $64 \%$ \\
\hline
\end{tabular}

Nota: Fuente cuestionario "Factores de riesgo psicosociales en población universitaria", 2012.

La Tabla 4 presenta que con respecto al factor codependencia, los resultados obtenidos entre estudiantes HADA y NO HADA también muestran similitud, casi dos terceras partes del estudiantado evidencia probabilidades de codependencia.

TABLA 5

Distribución del estudiantado participante según factor de riesgo autoestima y condición de ser HADA Y NO HADA. Sede Rodrigo Facio, Universidad de Costa Rica, 2012

\begin{tabular}{l|c|c|c|c|c|c}
\hline \multicolumn{1}{c}{$\begin{array}{c}\text { Factor } \\
\text { autoestima }\end{array}$} & N $\begin{array}{c}\text { Frecuenci } \\
\text { a relativa }\end{array}$ & N & $\begin{array}{c}c \\
\text { Frecuenci } \\
\text { a relativa }\end{array}$ & N & $\begin{array}{c}\text { Frecuencia } \\
\text { relativa }\end{array}$ \\
\hline Baja & 200 & $13 \%$ & 51 & $12 \%$ & 251 & $12 \%$ \\
\hline Moderada & 210 & $13 \%$ & 60 & $15 \%$ & 270 & $13 \%$ \\
\hline Normal o alta & $\begin{array}{c}118 \\
9\end{array}$ & $74 \%$ & 299 & $73 \%$ & 1488 & $74 \%$ \\
\hline
\end{tabular}

Nota: Fuente cuestionario "Factores de riesgo psicosociales en población universitaria", 2012.

En el factor de riesgo autoestima, los resultados de la Tabla 5 evidencian que casi las tres cuartas partes del grupo total se ubican con una autoestima normal o alta. Además, los resultados obtenidos entre estudiantes HADA y NO HADA son similares. 
TABLA 6

Distribución del estudiantado participante según factor de riesgo depresión y condición de ser HADA Y NO HADA. Sede Rodrigo Facio, Universidad de Costa Rica, 2012

\begin{tabular}{ccccccc}
\hline \multirow{2}{*}{$\begin{array}{c}\text { Factor } \\
\text { depresión }\end{array}$} & \multicolumn{2}{c}{ NO HADA } & \multicolumn{2}{c}{ HADA } & \multicolumn{2}{c}{ Total } \\
\cline { 2 - 7 } & $\mathrm{N}$ & $\begin{array}{c}\text { Frecuencia } \\
\text { relativa }\end{array}$ & $\mathrm{N}$ & $\begin{array}{c}\text { Frecuencia } \\
\text { relativa }\end{array}$ & $\mathrm{N}$ & $\begin{array}{c}\text { Frecuencia } \\
\text { relativa }\end{array}$ \\
\hline $\begin{array}{c}\text { No hay } \\
\text { sintomas }\end{array}$ & 527 & $33 \%$ & 103 & $25 \%$ & 630 & $31 \%$ \\
\hline Leve & 473 & $30 \%$ & 126 & $31 \%$ & 599 & $30 \%$ \\
\hline Moderado & 387 & $24 \%$ & 108 & $26 \%$ & 495 & $25 \%$ \\
\hline Severo & 212 & $13 \%$ & 73 & $18 \%$ & 285 & $14 \%$ \\
\hline
\end{tabular}

Nota: Fuente cuestionario "Factores de riesgo psicosociales en población universitaria", 2012

Según la Tabla 6, en el factor depresión, un 67 \% del estudiantado NO HADA presenta síntomas entre leves y severos. En la categoría síntomas severos de depresión se encontró una diferencia estadísticamente significativa en el estudiantado HADA, con respecto al grupo total.

TABLA 7

Diferencias entre los grupos HADA Y NO HADA según los factores de riesgo Prueba t student. Sede Rodrigo Facio, Universidad de Costa Rica, 2012

\begin{tabular}{lcccccc}
\hline \multicolumn{7}{c}{ GRUPOS } \\
\hline \multicolumn{7}{c}{ HADA } \\
\hline Media & DS & Media & DS & $T$ & Df \\
\hline Ansiedad & 11,7 & 4,5 & 11,3 & 4,7 & $-1,7$ & 2007 \\
\hline Codependencia & 4,0 & 1,5 & 4,0 & 1,4 & $-0,5$ & 2007 \\
\hline Autoestima & 32,5 & 5,9 & 32,7 & 5,8 & 0,8 & 2007 \\
\hline Depresión & 23,0 & 8,4 & 21,5 & 8,2 & $-3,4$ & 2007 \\
\hline
\end{tabular}

Nota: Fuente cuestionario "Factores de riesgo psicosociales en población universitaria”, 2012.

La Tabla 7 muestra que en los factores ansiedad, codependencia, autoestima y depresión no hay diferencias estadísticamente significativas entre el grupo HADA y el NO HADA.

\section{Discusión DE RESULTADOS}

En Costa Rica no se encuentran estudios sobre población universitaria con la condición de ser HADA; en otros países donde sí existen estudios en este tema, estos no son concluyentes en determinar la proporción de estudiantes con esa condición. Jiménez et al. (2011) indican que la proporción de HADA en investigaciones con muestras de estudiantes universitarios varía significativamente en la bibliografía. Al respecto, en este estudio se encontró que solamente un 20,5\% del grupo presenta características de la condición HADA, distribuido de manera muy similar entre hombres y mujeres (20\% hombres y $21 \%$ mujeres). Este porcentaje 
de estudiantes con características HADA es semejante a los hallazgos obtenidos en estudios con muestras relativamente pequeñas que se han realizado en los Estados Unidos (Jiménez et al., 2011).

El estudio evidencia que los factores de riesgo ansiedad, codependencia, baja autoestima y depresión se presentan de manera similar en el estudiantado HADA y NO HADA, puesto que no se encontraron diferencias estadísticamente significativas entre ambos grupos. De acuerdo con la distribución del estudiantado entre ser HADA y NO HADA, el resultado muestra que en ambos grupos las características que presenta la mayoría de la población son: no hay síntomas de ansiedad, con probabilidad de codependencia, una autoestima normal o alta y con síntomas de depresión entre leve, moderado y severo. Únicamente se encontró una diferencia estadísticamente significativa en los resultados del grupo HADA, es decir, este grupo de estudiantes muestra una mayor frecuencia (18\%) en los síntomas severos de depresión, comparados con el total de estudiantes. En relación con estos hallazgos, Jiménez et al. (2011) indican que hay estudios que no han encontrado diferencias entre los HADA y NO HADA en ajuste emocional y características de codependencia, lo cual coincide con los hallazgos de este estudio a excepción del factor de riesgo depresión.

En congruencia con los resultados, los factores de riesgo que requieren mayor atención en la población estudiantil de la Universidad de Costa Rica son la codependencia y la depresión. Así, al no existir diferencia entre los dos grupos de estudiantes (HADA y NO HADA), no es atribuible a esta condición la presencia de los factores de riesgo, es decir, no hay mayor vulnerabilidad en ninguno de los grupos por esa condición, situación que debe ser tomada en cuenta para la intervención con acciones de orientación.

El conjunto de características relacionadas con la codependencia incide en el desarrollo de las personas. Según los resultados de este estudio, un alto porcentaje de la población universitaria participante presenta rasgos de codependencia, que podrían afectar las relaciones que establecen con otras personas. Como lo señalan Valverde y Pochet (2006, p. 48), “...los hijos de adictos, incluso no consumidores, tienden a establecer vínculos afectivos y familiares con personas adictas o cuya personalidad concuerda con la de un adicto potencial". Por otra parte, las hijas e hijos de padres o madres con adicciones están expuestos a un funcionamiento que impacta la dinámica familiar, los roles, las relaciones intrafamiliares y la crianza, lo que puede repercutir en un impacto emocional. Esta población se integra al ámbito universitario con las repercusiones que tienen estas características de su sistema familiar, lo cual podría afectar su desarrollo académico y social en el aula universitaria y aumentar la probabilidad de que presenten síntomas de ansiedad y depresión. Sin embargo, una intervención desde la disciplina de orientación puede contribuir con el estudiantado, para que al ser consciente de su realidad, establezca estrategias y redes sociales que le permitan desarrollar habilidades para la toma de decisiones adecuadas en su vida.

La orientación también puede favorecer que el estudiantado universitario aprenda y potencie otras habilidades necesarias para establecer relaciones interpersonales sanas, incluidas las de pareja, en aras de que estas sean de satisfacción y estimulen su desarrollo personal y bienestar integral. Si una persona identifica herramientas que le favorecen en las interacciones que emprende, por ejemplo, la asertividad, estará satisfecha de los resultados, los cuales trascienden a todas sus esferas vitales. Pereira (2006) menciona que la orientación mediante su accionar contribuye al logro de objetivos educacionales mediante sus finalidades y métodos, los cuales, junto a los de la educación, convergen en el estímulo en el desarrollo de las áreas personal y social.

En cuanto al factor depresión, un 31\% no presenta síntomas, el $55 \%$ se ubicó en las categorías leves y moderadas; mientras un $14 \%$ presenta los síntomas de manera severa. La depresión en la actualidad es uno de los trastornos más comunes que afectan el desarrollo a lo largo de la vida. Con probabilidad, estudiantes con síntomas severos de depresión experimentan un bajo estado de ánimo, pérdida de interés, trastornos del sueño, disminución de la energía, malestares físicos, con consecuencias negativas que inciden, particularmente, en su papel como estudiante. Aunado a ello, las presiones que surgen del proceso de integración al ambiente universitario, las situaciones económicas, los problemas intrafamiliares y las dificultades de orden académico que experimenta el estudiantado resultan en altos niveles de estrés que 
inciden en el riesgo de que se presenten los trastornos como la depresión (Franck y Raedt, 2007; Vásquez y Blanco, 2009).

De manera que este escenario, en su amplitud, afecta directamente a la persona en su rol de estudiante, pues al presentarse un desequilibrio en el estado biopsicosocial, y al no experimentar bienestar físico y psicológico, se le dificulta el ajuste a las demandas académicas y se convierte en una dinámica que se presenta como un proceso circular constante.

La cotidianidad que vive el estudiantado en el contexto universitario, con las demandas académicas y los eventos relacionados con estas, podrían incidir en que se presenten síntomas de depresión, que aunque no alcancen un nivel severo, son causas de situaciones como la inactividad, la pérdida de iniciativa y una disminución o menor desempeño en las actividades de la vida diaria.

Según datos del Ministerio de Salud (2014), Costa Rica en el año 2013 reportó 41,576 casos nuevos de depresión, de estos el $74 \%$ corresponde a mujeres, lo que se asocia, por lo general, a situaciones de violencia intrafamiliar, desempleo, falta de acceso de medios económicos o endeudamientos; escenarios a los que podría estar expuesto el grupo universitario de estudiantes. Por ser la depresión una condición que ha venido en aumento en la población en general, es de esperar que esta situación se refleje también en la comunidad estudiantil universitaria y sus familias, por lo que es necesario que sea considerada en los servicios de apoyo que brinda la universidad.

En un estudio realizado por Arrivillaga, Cortés, Goicochea y Lozano (2004), con estudiantes de universidades, determinaron que la depresión está relacionada con variables como son los antecedentes personales y familiares de depresión. Esta conclusión cobra importancia en la investigación que aquí se reporta, al considerar que un 20,5\% de la población presenta características de ser HADA, es decir, son hijas e hijos de personas que presentan abuso de las bebidas alcohólicas. Esta característica podría significar que este grupo de estudiantes tiene mayores probabilidades de presentar síntomas de depresión, condición que posiblemente forma parte de los antecedentes familiares.

En relación con el factor autoestima, casi las tres cuartas partes de la población estudiantil presenta una autoestima entre normal y alta. Para el estudiantado HADA y NO HADA, la autoestima alta es, entonces, un factor protector que les hace sentirse capaces y con posibilidades, por lo tanto, de enfrentar las demandas del ambiente académico universitario, salvo como lo mencionan Cáceres, Salazar, Varela y Tovar (2006), que una alta autoestima en algunos casos puede provocar que las personas se sientan confiadas y asuman comportamientos de riesgo.

Aun cuando la mayoría del estudiantado participante en el estudio se ubicó en las categorías de normal y alta, hubo un porcentaje que corresponde a la autoestima baja (12\% de la población participante) y es precisamente para este grupo de estudiantes que la autoestima es un factor de riesgo, por cuanto se podría experimentar falta de confianza en las propias capacidades, así como para la resolución de las dificultades que presenta la vida universitaria y en general en su cotidianidad. En congruencia con el doble papel que cumple la autoestima, como factor protector y de riesgo, deben establecerse intervenciones en las que se fortalezca la autoestima del estudiantado como parte de un amplio proceso de autoconocimiento. Este auto aprecio permite que la persona confié en su potencial para lograr metas, en este caso, finalizar la carrera e ir concretando su proyecto de vida.

La autoestima es un proceso de construcción permanente que se ve influenciado por las experiencias que vive el estudiantado en su vida diaria. De Mezérville (2004) subraya que la autoestima es la percepción valorativa y confiada de sí mismo o de sí misma, que motiva a la persona para desenvolverse con autonomía y proyectarse satisfactoriamente en la vida.

En cuanto al factor ansiedad, los resultados del estudio mostraron que casi una tercera parte de la población participante presenta síntomas que van de leves a moderados. Martínez y Pérez (2014) mencionan que la ansiedad y el estrés tienen elementos comunes que dificultan su diferenciación; la ansiedad se utiliza para referirse a la experiencia interior de inquietud y desasosiego carente de objeto, donde el temor es impreciso, mientras que el estrés designa la sobrecarga emocional que se produce por una fuerza externa prolongada que 
pone a la persona en una condición de agotamiento; así cuando una persona enfrenta una situación que desde su propia percepción significa una amenaza, se convierte en evento estresor, generador de la sensación de ansiedad, pero no toda situación estresante lleva a la ansiedad, y mucho menos a un trastorno por ansiedad. La respuesta que la persona tenga ante los eventos de la vida va a depender de sus recursos personales y externos.

Por lo tanto, la presencia en el estudiantado participante de síntomas de ansiedad podría representar un factor de riesgo para el desempeño y el enfrentamiento de las demandas de la vida cotidiana y especialmente de las académicas en el contexto universitario. Se debe favorecer un manejo adecuado del estrés en la población estudiantil, porque un alto nivel puede contribuir a mayores niveles de ansiedad. Estrategias para la organización del tiempo, así como la relajación luego de los procesos arduos de estudio, son efectivas en el enfrentamiento adecuado de los niveles altos de estrés.

Finalmente, los resultados mostraron que prácticamente una quinta parte de la población participante manifiesta características HADA y los porcentajes obtenidos por hombres y mujeres fueron muy similares. Esto denota que este grupo de estudiantes podría provenir de familias en las que al menos uno de sus progenitores presenta abuso de bebidas alcohólicas, lo cual puede incidir negativamente en el desarrollo del potencial académico de sus miembros, incluyendo al estudiante o a la estudiante que forma parte de ese núcleo.

\section{Conclusiones}

Con respecto a los factores de riesgo -codependencia, depresión, ansiedad y baja autoestima- tomados en cuenta en este estudio, el grupo de estudiantes participantes en su mayoría muestran probabilidad de codependencia, con síntomas de depresión entre leves y moderados, con ausencia de síntomas de ansiedad y con una autoestima normal y alta.

Los factores de riesgo tomados en cuenta en la investigación se presentan de manera semejante, entre ambos grupos HADA y NO HADA, entre hombres y mujeres, y en el grupo en su totalidad, es decir, independientemente del factor el comportamiento, los datos tienden a ser similares. Es decir, los resultados muestran que no existen diferencias estadísticamente significativas apreciables en cuanto a los factores de riesgo estudiados en las poblaciones HADA y NO HADA, lo cual sugiere la necesidad de nuevas propuestas de estudios para profundizar en estos hallazgos.

Aun cuando el contexto familiar presente factores de riesgo, el ser parte de una comunidad universitaria es en sí mismo un factor protector, al ser la educación superior una posible fuente de desarrollo integral.

El estudiantado en su cotidianidad enfrenta una serie de demandas académicas y sociales que se extienden a lo largo de toda su formación profesional; esto requiere que la persona cuente con factores protectores que le permitan enfrentarlas e integrarse adecuadamente al contexto universitario. El estudio aporta información relevante de la población estudiantil de la Sede Rodrigo Facio, de la Universidad de Costa Rica, para comprender, prevenir y proponer acciones que contribuyan al enfrentamiento adecuado de los factores de riesgo.

Los resultados obtenidos evidencian la necesidad de fortalecer los servicios para el estudiantado universitario que se ofrecen para facilitar la integración al ambiente universitario, así como la prevención y atención de los factores incluidos en este estudio. Existe necesidad de trabajar de manera preventiva en una etapa temprana con las poblaciones para reducir el impacto individual y social que representa el desarrollo de factores de riesgo. Igualmente es importante que las personas profesionales que atienden los procesos de transición y desarrollo en la población estudiantil universitaria identifiquen de manera temprana la presencia de HADAS.

La orientación como profesión de ayuda a las personas está llamada a dar respuesta a las necesidades del estudiantado durante su permanencia en la universidad y a contribuir en el desarrollo de habilidades para 
hacer frente a los factores de riesgo, de manera tal que no se conviertan en una limitación para el éxito en sus estudios universitarios.

Es importante mencionar que los resultados de este estudio no pueden ser generalizados a la totalidad de la población universitaria; de ahí que para futuras investigaciones es recomendable replantear la selección de otro tipo de muestra y la aplicación del cuestionario en línea.

La investigación constituyó una valiosa experiencia de intercambio interuniversitario, con profesionales de Consejería de la Universidad de Puerto Rico, Recinto Río Piedras, quienes contribuyeron al enriquecimiento y diversificación de los procesos de investigación realizados en la Escuela de Orientación y Educación Especial, de la Universidad de Costa Rica.

\section{REFERENCIAS}

Alazraki, Y. (2006). La dinámica familiar en familias con un miembro alcohólico. Instituto Mejicano de la Pareja. Recuperado de http://www.ametep.com.mx/aportaciones/2006_abril_alazraki.htm

Arrivillaga, M., Cortés, C., Goicochea, V. y Lozano, T. (2004). Caracterización de la depresión en jóvenes universitarios. Revista Universitas Psychologica, 3(1). http://sparta.javeriana.edu.co/psicologia/publicaciones/ actualizarrevista/archivos/V3N102caracterizacion.pdf

Bejarano, J. (2012). Consumo de bebidas alcohólicas. Fascículo sobre alcohol. Serie 2 de 5. Consumo de Drogas en Costa Rica Encuesta Nacional 2010. Instituto sobre Alcoholismo y Farmacodependencia. Costa Rica. Recuperado de http://www.iafa.go.cr/investigaciones/epidemiologica/Fasciculo\%202.\%20Consumo\%20de \%20bebidas\%20alcoholicas15\%20de\%20junio012.pdf

Branden, N (1995). Los seis pilares de la autoestima. Barcelona: Paidós.

Cáceres, D., Salazar, I., Varela, M y Tovar, J. (2006). Consumo de drogas en jóvenes universitarios y su relación de riesgo y protección con los factores psicosociales. Revista Universitas Psychologica, 5(3). Recuperado de http:// pepsianc.bvsalud.org/pdf/up/v5n3/v5n3a08.pdf

Codependientes Anónimos. (s. f.). Manual de servicio de la fraternidad de codependientes anónimos. Recuperado de http://spanish.coda.org/index.cfm/programa-coda-relaciones-codependencia-codependientesanonimos/patrones-de-la-codependencia-2011/

De Mezérville, G. (2004). Ejes de salud mental. Los procesos de autoestima, dar y recibir afecto y adaptación al estrés. México: Trillas, S.A.

Gonzales, K. y Guevara, C. (2016). Autoestima en los universitarios ingresantes de la Facultad de Medicina d ella Universidad Nacional Mayor de San Marcos durante el año 2015. Revista de Investigación Apuntes Psicológicos, $1(1) .8-20$.

Franck, E., y De Raedt, R. (2007). Self-esteem reconsidered: Unstable self-esteem outperforms level of selfesteem as vulnerability marker for depression. [Repensar la autoestima: la autoestima inestable supera el nivel de autoestima como marcador de vulnerabilidad para la depresión] Behaviour Research and Therapy, 45, 1531-1541. doi:10.1016/j.brat.2007.01.003

Hernández, R., Fernández, C. y Baptista, O. (2008) Metodología de la investigación. México: Trillas.

Jiménez, M., Serra, J., Villafañe, A. y Jiménez, W. (2011). Hijos adultos de madres/padres alcohólicos y factores de riesgo psicológicos en estudiantes universitarios. Revista Actualidades Investigativas en Educación, 11(1), 1-20. DOI: https://doi.org/10.15517/aie.v11i1.10179

Manrique, L. (2017). Dinámica familiar del adolescente cuyos padres consumen alcohol y marihuana (Trabajo final de grado). Facultad de Enfermería y Rehabilitación, Universidad de La Sabana, Chía Cundinamarca, Colombia https://intellectum.unisabana.edu.co/bitstream/handle/10818/30383/Leidy \%20Marisol\%20Manrique\%20D\%c3\%adaz\%20\%28Tesis\%29.pdf?sequence=1\&isAllowed=y

Martínez, O. y Pérez, V. (2014). Ansiedad en estudiantes universitarios: Estudio de una muestra de alumnos de la Facultad de Educación. ENSAYOS. Revista de la Facultad de Educación de Albacete, 29-2. Recuperado de http://www.revista.uclm.es/index.php/ensayos 
Ministerio de Salud. (2014). Primeros datos oficiales sobre trastornos mentales permitirá incidir en la población y mejorar su salud mental. http://www.ministeriodesalud.go.cr/index.php/centro-de-prensa/noticias/662noticias-2014/694-primeros-datos-oficiales-sobre-trastornos-mentales-permitira-incidir-en-la-poblacion-ymejorar-su-salud-mental

Naranjo, M. (2007). Autoestima: Un factor relevante en la vida de la persona y tema esencial del proceso educativo. Revista Actualidades Investigativas en Educación, 7(3), 1-27. Recuperado de http://revista.inie.ucr.ac.cr/ uploads/tx_magazine/autoestima.pdf

Parada, J. (2010). La educación familiar en la familia del pasado, presente y futuro. Educatio Siglo XXI, 28(1), 17-40. Recuperado de http://www.revistas.um.es/educatio/article/view/109711

Pereira, M. (2006). Orientación educativa. San José, Costa Rica: EUNED.

Ramírez, M. y De Andrade, D. (2005). La familia y los factores de riesgo relacionados con el consumo de alcohol y tabaco en los niños y adolescentes (Guayaquil Ecuador). Rev Latino-am Enfermagem, 13. Recuperado de http:// www.scielo.br/pdf/rlae/v13nspe/v13nspea08.pdf

Riveros, M., Hernández, H., Rivera, J. (2007) Niveles de depresión y ansiedad en estudiantes universitarios de Lima metropolitana. Revista Investigación Psicológica, 10(1), 91-102. Recuperado de http://dialnet.unirioja.es/ servlet/articulo?codigo $=2348649$

Rodríguez, P. (2008) Ansiedad y sobreactivación: Guía práctica de entrenamiento en control respiratorio. España: Editorial Desclée de Brouwer, Recuperado de http://site.ebrary.com/lib/sibdilibrosp/Doc? id=10479340\&ppg $=20$

Sierra, J., Ortega, V. y Zubeidat, I. (2005) Ansiedad, angustia y estrés: Tres conceptos a diferenciar. Editorial: Red Revista Mal-estar E Subjetividade. Recuperado de http://site.ebrary.com/lib/sibdilibrosp/docDetail.action? $\operatorname{doc} \mathrm{ID}=10105169 \& \mathrm{p} 00=$ ansiedad

Valverde, L. y Pochet, J. (2006). Drogadicción: Hijos de la negación. Revista de Ciencias Sociales. Universidad de Costa Rica. Recuperado de http://site.ebrary.com/lib/sibdilibrosp/Doc?id=10110811\&ppg=8

Vásquez F. y Blanco, V. (2009). Prevalence of DSM-IV major depression among Spanish university students. [La prevalencia de depresión mayor del DSM-IV entre los estudiantes universitarios españoles]. Journal of American College Health, 57(2), 165-171.

\section{Notas}

[1] Orientadora, con un Doctorado en Educación, con énfasis en Mediación Pedagógica, Actualmente labora como docente de la Escuela de Orientación y Educación Especial, es investigadora activa en el Instituto de Investigaciones en Educación y docente en la Maestría en Educación con énfasis en Orientación laboral. Es lectora y directora de trabajos finales de graduación. Anteriormente laboró por casi 15 años con el Ministerio de Educación Pública, como orientadora en Equipos Interdisciplinarios y con Departamentos de Orientación de Secundaria.

[2] Orientadora y con un posgrado en Evaluación Educativa de la Universidad de Costa Rica. Profesora asociada, jubilada de la Escuela de Orientación y Educación Especial. Directora de trabajos finales de graduación.

\section{BY-NC-ND}

